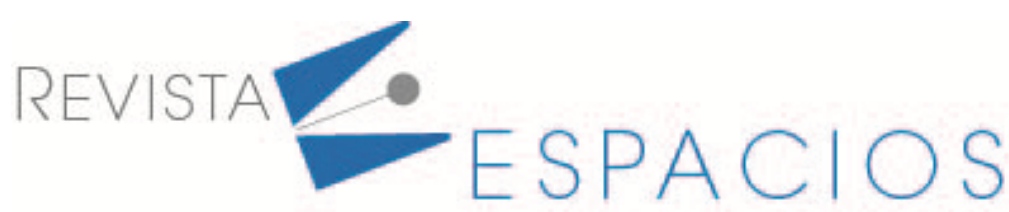

\title{
Análisis de la competitividad exportadora de los productores de mezcal de Oaxaca y Michoacán (México) al mercado de los Estados Unidos
}

\section{Analysis of the export competitiveness of mezcal producers from Oaxaca and Michoacán (Mexico) to the United States market}

\author{
SALINAS GARCIA Esteban ${ }^{1}$ \\ ALCARÁZ VERA Jorge Víctor ${ }^{2}$
}

\begin{abstract}
Resumen El objetivo de esta investigación fue conocer las variables que incidieron en la competitividad exportadora de las MIPYMES productoras de mezcal de los estados de Oaxaca y Michoacán, México, hacia el mercado de los EE.UU. La metodología consistió en la aplicación de un cuestionario de estructura cerrada. Se comprobó que la Productividad y el uso de Tecnología tienen una incidencia importante en la competitividad de las empresas productoras de mezcal, no así la variable Estructura Organizacional.

Palabras clave: competitividad, productividad, estructura organizacional, tecnología
\end{abstract}

\begin{abstract}
The objective of this research was to know the variables that influenced the export competitiveness of the mezcal-producing MSMEs from the states of Oaxaca and Michoacán, Mexico, towards the US market. The methodology consisted in the application of a questionnaire of closed structure. It was found that Productivity and the use of Technology have an important impact on the competitiveness of mezcal-producing companies, but not the Organizational Structure variable.

Key words: competitiveness, productivity, organizational structure, technology
\end{abstract}

\section{Introducción}

Las Mipymes de la industria del mezcal se han visto limitadas en su crecimiento durante las últimas décadas, así como en su producción y capacidad de exportación, por ello la importancia de este estudio para conocer las causas que impiden una mayor competitividad exportadora de los productores del mezcal en las regiones objeto de estudio. Existen estudios relacionados con la comercialización, la productividad, la tecnología, etc. sin embargo, se carecen de estudios relacionados con la competitividad exportadora de los productores de mezcal.

\footnotetext{
${ }^{1}$ Graduado de la maestría en Ciencias en Negocios Internacionales del Instituto de Investigaciones Económicas y Empresariales de la Universidad Michoacana de San Nicolás de Hidalgo. Michoacán, México. Correo electrónico de contacto: 0732565b@umich.mx.

2 Profesor Investigador del Instituto de Investigaciones Económicas y Empresariales de la Universidad Michoacana de San Nicolás de Hidalgo, Michoacán, México. Correo electrónico de contacto: talcarazv@hotmail.com
} 
Una de las limitaciones en la realización de este trabajo de investigación estuvo relacionado con la confianza de los productores para responder a los cuestionarios aplicados.

\section{Marco teórico}

\subsection{Principales teorías de la competitividad}

Sin duda la competitividad económica se halla en un estado de flujo constante, de ahí la pregunta: ¿a qué se debe la ventaja competitiva de la que gozan algunas naciones? Algunos expertos refieren que los factores fundamentales son los costos de la mano de obra, las tasas de interés, el tipo de cambio y las economías de escala; otras de las maneras en que las empresas logran una mayor competitividad consiste en hacer obsoletas las innovaciones; es decir, desarrollan un nuevo producto que reemplace al anterior, lo que implica el que sigan haciendo innovaciones mejorando constantemente sus procesos operativos y administrativos (Rugman, 2001).

¿Qué deben hacer los países si quieren tener posiciones sólidas de inversión y comercio internacional? Son tres las áreas en las que pueden tener su fortaleza. Competitividad económica, Influir en las regulaciones comerciales para que el resto de las naciones abran sus fronteras a los bienes y servicios del exterior y estar dispuestos a comprar y vender en el comercio mundial y finalmente deben desarrollar una orientación global (Rugman, 2001).

Asimismo, Ibarra (2017) menciona a la competitividad como la posibilidad que tienen los ciudadanos para elevar su nivel de vida, que está en función de la productividad con la que se utilizan los recursos nacionales, el producto por unidad de trabajo o el capital utilizado. Por otro lado, la competitividad se alcanza elevando la productividad en los negocios existentes o involucrarse en negocios con mayor productividad. Menciona que en la competitividad a nivel micro o empresarial se pueden distinguir ocho dimensiones, las cuales de manera combinada determinan qué tan competitiva es una empresa, así como el grado de éxito que ésta presenta respecto al resto del mercado. Dichas dimensiones son las siguientes:

a) Planeación estratégica: se refiere a si las organizaciones tienen objetivos y metas a plazo, políticas para su cumplimiento y seguimiento.

b) Producción y operaciones: Su aportación radica en la complejidad de los procesos de producción, uso de herramientas modernas de producción, certificaciones, flexibilidad en procesos productivos, planeación de materias primas e insumos, desarrollo de nuevos productos, manejo de inventarios, entre otros factores.

c) Aseguramiento de la calidad: Se determina el nivel de aplicación de las normas de calidad, programas para enfrentar contingencias, grupos de trabajo al respecto, certificaciones.

d) Comercialización: consiste en el análisis de las políticas de venta, definición del mercado meta, estudios de mercado, satisfacción del cliente, uso de estrategias de mercadeo, vías de distribución, formas de pago, relación con clientes y proveedores.

e) Contabilidad y finanzas: Se ocupa de atender todo lo relacionado con los aspectos operativos y administrativos, pago de impuestos, etc.

f) Recursos humanos: parte de que la competitividad de una organización se relaciona con el adecuado uso del recurso humano, es esencial contar con un proceso riguroso de selección y contratación, programas de capacitación y adiestramiento, análisis de las causas de la rotación laboral, clima laboral y los programas para remediarlos, sistemas de compensaciones, cumplimiento de aspectos de seguridad e higiene industrial.

g) Gestión ambiental: las nuevas exigencias en esta materia son de mayor relevancia para los consumidores, las empresas deben ser competitivas y a la vez responsables con el medio ambiente. 
h) Sistemas de información: Es un área importante para que los negocios alcancen un alto nivel de competitividad en el mercado.

La competitividad relacionada con la exportación de acuerdo con Pérez (2007), es planteada como la eficiencia de cada uno de los negocios ante el resto de los negocios del mismo giro en el mercado. Para Saavedra y Tapia, (2011) los elementos más importantes a considerar en la competitividad de un negocio son: producción, rentabilidad, sector, participación en el mercado, infraestructura, entre otras.

Paralelamente, de acuerdo con el enfoque sistémico propuesto por Esser, Hilebrand, Messner y Meyer (1994), son cuatro esferas las que señalan el nivel de competitividad de una MIPYME en un país industrializado o emergente:

- Micro: adecuaciones internas de la empresa y su entorno.

- Meso: estructura institucional eficiente para una buena interacción con actores externos de la empresa.

- Macro: estabilidad relacionada con las políticas y reformas que el gobierno implementa, así como acciones, las cuales afectan a la operatividad de la empresa o su mercado.

- Meta: estructura social y política de la sociedad entera que conducen a su desarrollo.

La tendencia en el comercio internacional exige una reestructuración para las empresas, como mejoras en sus procesos de producción que no sólo consideran a la tecnología o los insumos usados, se precisa integrar a los procesos indirectos que aumenta el nivel de competitividad. Uno de ellos es la Responsabilidad Social Empresarial (RSE).

Según Porter (2015) la competitividad está directamente relacionada con la productividad, bajo este supuesto todas las empresas deben contar con una alta productividad en sus procesos operativos y administrativos para ser considerados como empresas eficientes en el mercado.

Se puede observar en la Tabla 1, cómo México ha perdido terreno en competitividad frente a países de la región como Chile y Perú, en pilares como eficiencia económica, eficiencia del gobierno, eficiencia de los negocios e infraestructura.

Tabla 1

Resultados del Ranking de Competitividad y de

Pilares de los Países Latinoamericanos: 2020.

\begin{tabular}{lcccccccccc}
\hline Países & \multicolumn{2}{c}{$\begin{array}{c}\text { Ranking } \\
\text { General }\end{array}$} & \multicolumn{3}{c}{$\begin{array}{c}\text { Desempeño } \\
\text { Económico }\end{array}$} & \multicolumn{2}{c}{$\begin{array}{c}\text { Eficiencia del } \\
\text { Gobierno }\end{array}$} & \multicolumn{2}{c}{$\begin{array}{c}\text { Eficiencia de } \\
\text { los negocios }\end{array}$} & \multicolumn{2}{c}{ Infraestructura } \\
\hline Chile & 67.08 & 38 & 51.91 & 50 & 72.13 & 20 & 55.11 & 37 & 44.54 & 45 \\
Perú & 54.87 & 52 & 51.90 & 51 & 53.65 & 40 & 41.97 & 50 & 27.33 & 60 \\
México & $\underline{\mathbf{5 4 . 8 0}}$ & $\mathbf{5 3}$ & $\underline{\mathbf{5 8 . 4 8}}$ & $\mathbf{3 8}$ & $\underline{\mathbf{4 0 . 0 2}}$ & $\underline{\mathbf{5 5}}$ & $\underline{\mathbf{4 3 . 8 0}}$ & $\underline{\mathbf{4 8}}$ & $\underline{\mathbf{3 2 . 2 5}}$ & $\underline{\mathbf{5 7}}$ \\
Colombia & $\mathbf{5 2 . 1 5}$ & $\mathbf{5 4}$ & $\mathbf{5 1 . 7 4}$ & $\mathbf{5 2}$ & $\mathbf{3 9 . 9 5}$ & $\mathbf{5 6}$ & $\mathbf{3 9 . 2 5}$ & $\mathbf{5 2}$ & $\mathbf{3 3 . 0 4}$ & $\mathbf{5 6}$ \\
Brasil & 49.63 & 56 & 47.57 & 56 & 24.59 & 61 & 44.44 & 47 & 37.28 & 53 \\
Argentina & 38.06 & 62 & 36.50 & 60 & 10.77 & 63 & 21.19 & 62 & 39.15 & 52 \\
Venezuela & 25.37 & 63 & 8.39 & 63 & 15.72 & 62 & 26.20 & 60 & 6.54 & 63 \\
\hline \multicolumn{8}{c}{ Fuente: Elaboración propia con base en FEM (2020) }
\end{tabular}

\subsection{Principales teorías de la Productividad}

La literatura refiere que Quesnay (1766), fue el primero en utilizar el término de productividad, pero no fue sino hasta 1883 cuando Littré expuso que la productividad es la facultad de producir algo; cuando se discute el concepto de productividad es común que se le relacione o se le considere sinónimo de producción, productividad, eficiencia y efectividad (Alvarado y Sánchez, 2019). 
Por su parte Pritchard citado en Alvarado y Sánchez (2019), señala cinco perspectivas a través de las cuales se puede estudiar la productividad.

1. Perspectiva de la Economía: Concibe a la productividad como la cantidad de productos generados dividido entre la cantidad de insumos asociados y/o entradas como lo son el trabajo, el capital, los productos intermedios adquiridos y el tiempo.

2. Perspectiva de la Ingeniería: La productividad es equiparada con la eficiencia de operación, basada en la comparación de la energía como el principal insumo y la cantidad de trabajo aplicada para generar los productos de salida, considérese la medición de unidades producidas por Kilowatt-hora, Horas-HombreHoras-Máquina entre otras.

3. Perspectiva de la Contabilidad: La productividad se enfoca en el desempeño financiero de la organización.

4. Perspectiva de la Administración: Bajo este enfoque a la productividad se considera un concepto de orden complejo debido que está en función de medir y evaluar factores tales como la calidad, la cantidad de recursos y productos aplicados, interferencias operativas, los retornos financieros y el ausentismo laboral.

5. Perspectiva de la Psicología Organizacional: Desde este punto de vista, la productividad está relacionada principalmente con la eficacia y la eficiencia del factor humano derivado del desarrollo de sus funciones y tareas.

Las ventajas para una empresa por contar con una mejor productividad son:

a) Mayores utilidades, ya sea por mayor margen de utilidad, o por un mayor volumen de ventas.

b) Ingresos mayores para los empleados.

c) Mayor margen de seguridad en el mercado, es decir, mayor competitividad.

d) Inmejorables oportunidades de expansión.

e) Prestigio social.

Kopelman (1988) citado por Alvarado y Sánchez (2019), menciona que la productividad es afectada por muchos factores como la cantidad y la complejidad técnica de los equipos y bienes de capital, la calidad y la disponibilidad de las materias primas, el volumen de las operaciones, la habilidad, la motivación y las actitudes de los empleados, el flujo de la organización y la competencia gerencial. Se refiere a los resultados que consiguen las organizaciones en la medida en que se dé una relación entre los factores que intervienen para lograrlos, por lo que puede señalarse que el incremento de la rentabilidad de la empresa depende de las mejoras de la productividad; de manera que tanto la rentabilidad y la productividad deben planificarse y aplicarse continuamente en el personal, ya que si una organización cuenta con el compromiso del factor humano hacia el logro de los objetivos, se hará posible el incremento de la productividad.

Desde la economía, Adam Smith afirma que el producto anual de la tierra y del trabajo de la nación sólo puede incrementarse por dos vías: un adelanto en las facultades productivas del trabajo útil que dentro de ellas se mantiene, o el aumento en la cantidad del trabajo (Jaimes y Ludym 2018).

\subsection{Aproximaciones teóricas de la estructura organizacional}

El análisis de la estructura organizacional se enmarca dentro de la Teoría de la Contingencia Estructural, la cual surge para mostrar, que no existe una única forma de estructuración, pues la división del trabajo y su 
coordinación, dependerán de diversos factores y de las condiciones situacionales en las cuales la organización opera (Idárraga, 2012).

Si las organizaciones son los ámbitos en que los recursos se transforman en resultados, estos recursos humanos y materiales que cuestan dinero e incluyen tecnologías, deben ser empleados de manera que se logren los resultados necesarios con calidad, costo y oportunidad aceptables, es decir, de manera compatible con la supervivencia organizacional (Hintze, 1999).

La estructura organizativa, es el instrumento técnico final que permite completar el proceso de asignación de responsabilidad hasta el grado de detalle necesario para el uso adecuado de los recursos en la ejecución de las actividades. Este detalle debe llegar hasta dos niveles: el de las unidades organizativas o centros de responsabilidad, y en una segunda instancia, el de los puestos de trabajo concretos que ocupará cada persona (Hintze, 1999).

Toda empresa requiere necesariamente de una estructura organizacional o una forma de organización (Tabla 2) de acuerdo a sus necesidades y en función de su tamaño, por medio de la cual se pueden ordenar actividades, los procesos y su funcionamiento como empresa (Mintzberg, 1993).

Tabla 2

Tipos de estructuras y clases

de unidades organizativas.

\begin{tabular}{|l|l|l|l|}
\hline \multirow{2}{*}{\multicolumn{2}{|l|}{}} & \multicolumn{2}{c|}{ TIPO DE ESTRUCTURA } \\
\cline { 3 - 4 } & \multicolumn{1}{c|}{ VERTICAL } & \multicolumn{1}{c|}{ HORIZONTAL } \\
\cline { 3 - 4 } & \multicolumn{1}{|c|}{$\begin{array}{l}\text { Unidades con estructura prevista para } \\
\text { gestiones permanentes }\end{array}$} & $\begin{array}{l}\text { Unidades con estructura prevista } \\
\text { para proyectos o programas }\end{array}$ \\
\hline UNIDAD & $\begin{array}{l}\text { LíNEA } \\
\text { Unidades con } \\
\text { responsabilidad } \\
\text { principal de } \\
\text { producción externa } \\
\text { de la institución }\end{array}$ & $\begin{array}{l}\text { Unidades de la conducción general } \\
\text { institucional y responsables de } \\
\text { producir los resultados que la } \\
\text { institución entrega a terceros en forma } \\
\text { sistemática y rutinaria }\end{array}$ & $\begin{array}{l}\text { Unidades de proyectos y } \\
\text { programas vinculados a producción } \\
\text { de resultados para terceros }\end{array}$ \\
\cline { 2 - 4 } & $\begin{array}{l}\text { STAFF } \\
\text { Unidades con } \\
\text { responsabilidad } \\
\text { principal de apoyo a } \\
\text { la producción externa } \\
\text { de la institución }\end{array}$ & $\begin{array}{l}\text { Unidades de planificación, } \\
\text { administración, apoyo legal, recursos } \\
\text { humanos, mantenimiento, logística y } \\
\text { otros cuyos aportes mantienen la } \\
\text { organización para que se produzcan } \\
\text { los resultados externos }\end{array}$ & $\begin{array}{l}\text { Unidades de proyecto y programas } \\
\text { propia organización, tales como } \\
\text { reorganizaciones, reingenierías, } \\
\text { modernizaciones informáticas, } \\
\text { nuevas instalaciones y otros }\end{array}$ \\
\hline
\end{tabular}

Fuente: Elaboración propia con base en Hintze (1999).

La Organización para la Cooperación y el Desarrollo Económico (OCDE) citada por Jinémez (2006), refiere tres capacidades básicas, para elevar la productividad y competitividad de las empresas y las economías, generando las condiciones para el desarrollo; éstas son: capacidad de innovación, capacidad de adaptación y capacidad de aprendizaje.

\subsection{La tecnología y las MIPYMES}

La innovación tecnológica desde hace muchos años ya lo asociaban con la competitividad, en su momento Adam Smith, David Ricardo, Karl Marx, Weber, Khun y Schumpeter, señalaban la importancia de esta relación. La actual dinámica para la generación de riqueza, requiere capacidades científicas y tecnológicas, como generadores de productividad, competitividad y desarrollo económico (Chávez, 2012). 
Para Nagles (2007) afirma que la innovación es la capacidad de la empresa para generar soluciones ingeniosas, creativas y rentables de manera que atienda las necesidades, expectativas y demandas de los consumidores, mercados y sociedad en general.

Las capacidades tecnológicas de una empresa se transforman en capacidades distintivas cuando se realizan mejor que los competidores, y cristalizan, siguiendo la terminología de Prahalad y Hamel (1991) citados en Claver, (2000), en competencias esenciales y además, permiten el acceso a múltiples mercados y generan mucho valor para el cliente.

Las tecnologías son combinatorias en el sentido de que ninguna suele ser empleada aisladamente, es decir, cualquier producto fabricado por una empresa o cualquier proceso productivo empleado por ésta, resulta de la combinación de un número determinado de tecnologías (Claver, 2000).

El nuevo enfoque de la competitividad genera cambios en el mercado actual, por lo tanto, se reducirá el ciclo de vida de los productos por la apuesta a la innovación constante, además de un continuo diseño de los productos por el aprovechamiento de la tecnología y una entrada de agentes externos al mercado al momento de aumentar la productividad (Matta, 2015).

La siguiente tabla muestra las revoluciones tecnológicas que se han producido a lo largo de la historia económica, con la fortuna de ser testigos de la transformación de tecnologías intensivas en capital y energía hacia tecnologías intensivas en información, cambio que está modificando radicalmente las condiciones técnicas de producción, la cantidad, calidad y utilidad de los bienes de que dispone la sociedad e inclusive la relación humana y al propio tiempo, las maneras de concebir y practicar la competitividad, por cuanto la dinámica del nuevo paradigma productivo en un proceso de destrucción creativa acorta la vida de los productos, cuya consecuencia es una competencia en ideas traducidas en una innovación constante y permanente que se constituye en el núcleo básico de la competitividad.

Tabla 3

Cinco revoluciones tecnológicas en 230 años.

\begin{aligned} & \hline Año \\ & \hline 1771 Revolución industrial \\ & 1829 Época del hierro, la máquina de vapor y el ferrocarril \\ & 1875 Época del acero y la ingeniería pesada \\ & 1908 Época del petróleo, del automóvil y la producción en masa \\ & 1971 Época de la informática y las telecomunicaciones \\ & $20 ? ?$ Época de biotecnología, nanotecnología y bioelectrónica \\ & \hline\end{aligned}

Fuente: Elaboración propia con base en Peñaloza (2007).

Las Tecnologías de la Información y Comunicación (TIC) constituyen una herramienta de soporte en su camino a la competitividad, siendo sustancial la percepción que los empleados tienen hacia la tecnología y su disposición para formarse en medios tecnológicos, que les ayude a elevar su nivel de productividad y eficiencia, por el contrario, si no se tiene la preparación para utilizarla, el trabajo se vuelve complicado y bajan los niveles de eficacia. Por ello, es importante que las empresas estén preparadas tecnológicamente para enfrentar los retos que impone coexistir en un mundo globalizado (Ríos, 2014).

\section{Marco referencial}

El mezcal, en su definición más antigua y acertada, es una bebida espirituosa destilada a partir de la fermentación exclusiva de azúcares provenientes de cualquier especie o variedad de agave (Saldaña, 2012). El estado de Oaxaca tiene la mayor biodiversidad de agaves de México y del mundo, por lo que no es raro que sea justamente 
ahí donde se aprovechen la mayor cantidad de especies silvestres. La especie cultivada más popular para hacer mezcal es el agave espadín.

En el caso de los estados de Michoacán, Hidalgo, Estado de México, Puebla y Guerrero, la mayor parte de su población se ha dedicado a actividades agrícolas desde tiempos anteriores a la colonia española. Los agaves cultivados son sembrados a partir de hijuelos, semillas o bulbillos florales en tierras de cultivo o laderas de monte (Saldaña, 2012).

Proceso de producción del mezcal:

- Cocimiento: El cocimiento de maguey puede hacerse usando tecnologías distintas y tiene como objetivo que los azúcares de la piña se transformen en azúcares fermentables.

- Molienda: Es el proceso de desgarrar las fibras de las piñas para extraer su jugo cocido, algunos separan el bagazo o fibra de las piñas del jugo y otros no.

- Fermentación: Los jugos y/o fibras del agave se ponen en tinas, se le suele agregar agua, levaduras y microorganismos transforman los jugos en mosto fermentado.

- Destilación: En la destilación los mostos fermentados, que contienen alcohol y una enorme cantidad de otros compuestos que integrarán el mezcal, los cuales son sometidos a un proceso de calentamiento y condensación en al menos dos etapas. En la primera se obtiene el ordinario y en el segundo se consigue el mezcal final (Saldaña, 2012).

Una de las principales bebidas que se comenzó a producir en México es el mezcal, el cual surgió a partir de la mezcla de la tradición prehispánica en cuanto al uso del maguey y la técnica de destilación importada por los españoles, actualmente se considera que existen más de doscientas especies. Si bien se han identificado diversos tipos de producción de mezcal tales como el artesanal, tradicional y moderna, la producción artesanal predomina entre los habitantes del estado de Oaxaca y Michoacán.

Desde mediados del siglo XX y de lo que va del XXI, se puede hablar de dos clasificaciones de los productores: los artesanales y los industrializados, esta última enfocada principalmente en la generación de utilidades y en la expansión del capital, por lo cual, a través de la tecnología, las regulaciones y las estrategias comerciales permiten la reducción de los costos y el aumento de las utilidades. Esto porque se requiere mayor inversión de capital para la tecnificación de los procesos productivos (Placencia; Peralta, 2018).

Uno de los retos presentes para la comercialización del mezcal es la falta de un desarrollo integral de promoción y publicidad. Por lo tanto, como lo menciona Catarina (2019), un crecimiento de la venta del mezcal, ya sea en el mercado local, nacional e internacional, generará un efecto multiplicador en la industria, ya que se podrá incrementar de manera exponencial la venta de los insumos que se requiere para el envasado, etiquetado, etc.

México cuenta con la Denominación de Origen (DO) del mezcal, la cual protege la propiedad intelectual de 39 municipios de Durango, 1 de Guanajuato, 81 de Guerrero, 570 de Oaxaca, 58 de San Luis Potosí, 11 de Tamaulipas y 58 de Zacatecas. El estado más recientemente incorporado al plano nacional de productores reservados de mezcal es Michoacán con 29 municipios, incluido en el año 2012 (Pérez, 2016). En el contexto productivo, de las 111,420 hectáreas sembradas en 2016 , el $89.78 \%$ de la superficie se encuentra mecanizada, $51.89 \%$ cuenta con tecnología aplicada a la sanidad vegetal, mientras que $67.78 \%$ del territorio sembrado con este cultivo contó con asistencia técnica. Por otro lado, el $98.65 \%$ de la producción es de temporal.

Como se puede observar en la gráfica 1, los principales países importadores de mezcal son: EE. UU en primer lugar, en seguida vienen Alemania, Japón y Reino Unido principalmente. 
Gráfica 1

Principales importadores mundiales de mezcal

(millones de litros)

\begin{tabular}{|c|c|c|}
\hline Estados Unidos & & 131.402 \\
\hline Alemania & 73.285 & \\
\hline Japón & 66.039 & \\
\hline Reuno Unido & 37.043 & \\
\hline España & 36.003 & \\
\hline Panamá & 26.392 & \\
\hline Rusia & 22.506 & \\
\hline Îndia & 18.747 & \\
\hline Dinamarca & 15.871 & \\
\hline Francia & 13.018 & \\
\hline
\end{tabular}

Fuente: SAGARPA, (2017).

En la gráfica 2 podemos observar que México es el principal país exportador de mezcal en el mundo; en consecuencia, también es el mayor productor a nivel mundial, le siguen Corea del Sur y Países Bajos, entre otros, sin embargo, nuestro país destaca en la exportación de este producto.

Gráfica 2

Principales exportadores mundiales de mezcal (millones de litros)

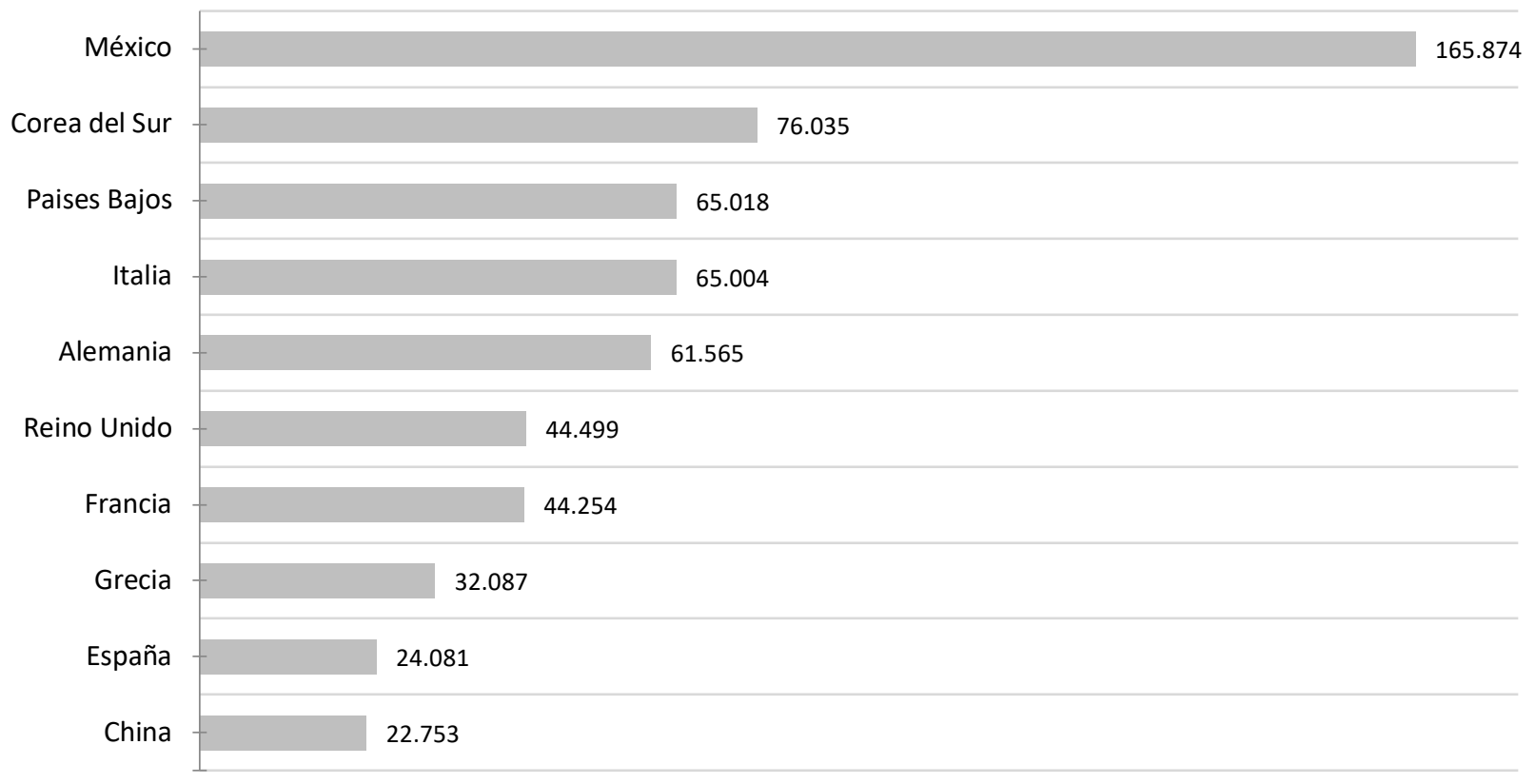

Fuente: SAGARPA, (2017)

En el estado de Michoacán, el 26\% de los municipios cuentan con la DO del mezcal, para gran parte de estos municipios su actividad principal es la producción y comercialización de esta bebida, por lo que se requiere construir una estrategia de competitividad conjunta gobierno-estado-empresa para su mejora que incluya la 
certificación del producto encaminada hacia una competitividad sustentable. Estos microempresarios han realizado sus procesos de producción en forma empírica, pasando los conocimientos de padres a hijos de manera tradicional sin documentar las actividades realizadas; esto les permite tener un bagaje cultural desarrollado, pero una muy baja capacidad de normalizar estos procesos. Desde hace cinco años, la zona mezcalera de Michoacán obtuvo la DO; sin embargo, su comercialización y venta no se ha incrementado debido a la baja competitividad normativa de la industria mezcalera en comparación con otras entidades como Oaxaca o San Luis Potosí (Tabla 4).

El 22 de noviembre de 2012, se modificó la Declaración General de Protección de la DO mezcal, publicada en el Diario Oficial de la Federación (DOF) el 28 de noviembre de 1994, para incluir dentro del listado que comprende el territorio de la misma el corredor geográfico del mezcal, los siguientes municipios pertenecientes al estado de Michoacán: Acuitzio, Aguililla, Ario, Buenavista, Charo, Chinicuila, Coalcomán de Vázquez Pallares, Cotija, Cojumatlán de Régules, Erongarícuaro, La Huacana, Tacámbaro, Turicato, Tzitzio, Hidalgo, Salvador Escalante, Morelia, Madero, Queréndaro, Indaparapeo, Tarímbaro, Tancítaro , Los Reyes, Tepalcatepec, Sahuayo, Marcos Castellanos, Jiquilpan, Venustiano Carranza y Vista Hermosa, los cuales utilizan principalmente Maguey Cupreata como materia prima (Chávez M., 2016).

Tabla 4

Producción de mezcal certificado por los principales estados

\begin{tabular}{lcccccccc}
\hline $\begin{array}{c}\text { Eslabones de la cadena } \\
\text { productiva }\end{array}$ & Oaxaca & Guerrero & Zacatecas & Durango & $\begin{array}{c}\text { S. Luis } \\
\text { Potosí }\end{array}$ & Guanajuato & Tamaulipas & Michoacán \\
\hline Productor de agave & 212 & 24 & 4 & 9 & 2 & 4 & 3 \\
\hline Productor de mezcal & 332 & 43 & 20 & 26 & 7 & 4 & 4 & 1 \\
\hline Envasador & 135 & 6 & 19 & 15 & 6 & 6 & 2 & 1 \\
\hline Comercializador & 155 & 7 & 21 & 13 & 6 & 5 & 1 \\
\hline Prestador de servicios & 2 & 0 & 0 & 0 & 0 & 0 & 0 \\
\hline
\end{tabular}

Fuente: Elaboración propia con base en SAGARPA (2015).

La Fundación PRODUCE Oaxaca (2007) citada en Bautista (2015), destaca los indicadores sobre el desarrollo agroindustrial del mezcal en Oaxaca, resaltando la exportación del 30\% del volumen de mezcal producido y envasado hacia la Comunidad Económica Europea (CEE).

\section{Marco metodológico}

Esta investigación es de orden explicativo y de corte mixto preponderantemente cuantitativo, dado que se buscó conocer la incidencia que tienen las variables productividad, tecnología y estructura organizacional en la competitividad exportadora de las MIPYMES productoras de mezcal en los estados objeto de estudio; para ello se realizó el trabajo de campo con la herramienta más indicada para investigaciones de esta naturaleza, se aplicó una muestra de 100 cuestionarios (Anexo 1) con un total de 39 preguntas cada uno, elaborados bajo una escala tipo Likert, una vez que se obtuvieron los datos se procesaron en el programa SPSS, aplicando la prueba de coeficiente de correlación de Pearson, lo que permitió conocer el grado de incidencia que tiene cada una de las variables independientes sobre la variable dependiente, objetivo principal de este trabajo. Es importante mencionar que no existen estudios previos relacionados con este tema de investigación.

\section{Análisis, discusión e interpretación de resultados}

Esta investigación surge por la inquietud de conocer las causas que impiden y limitan la posibilidad para que los productores de mezcal ubicados en los estados de Oaxaca y Michoacán, México, exporten sus productos al mercado internacional, para ello se seleccionaron tres variables independientes: productividad, tecnología y 
estructura organizacional, que de acuerdo a Ibarra (2017) y Catarina (2019), son algunas de las variables que inciden de manera importante en la competitividad de una MIPYME; no significa que sean las únicas, sin embargo para efectos de este tema de investigación se eligieron estas variables.

Se aplicó la prueba Alfa de Cronbach a los ítems del cuestionario en el que se obtuvo el valor de .762, lo que significa que es altamente confiable para la realización del trabajo de investigación.

Tabla 5

Prueba alfa de Cronbach

\begin{tabular}{cc}
\hline \multicolumn{2}{c}{ Estadísticas de fiabilidad } \\
\hline Alfa de Cronbach & N de elementos \\
\hline .762 & 10 \\
\hline
\end{tabular}

Fuente: Elaboración propia con base en SPSS (2020).

De acuerdo a lo que se observa en la tabla 6, se puede afirmar que la productividad es una variable que incide de manera importante en la competitividad de una empresa del sector mezcalero, ya que en primer lugar en su mayoría pertenecen a micro empresas, es decir, el $66.7 \%$ de los encuestados y en menor medida a pequeñas y medianas empresas (PYMES), esto va en sintonía con el número de hectáreas que producen: el 38.3\% de los encuestados producen entre 6 y 8 hectáreas de maguey para la obtención del mezcal y sólo el 11.7\% de los encuestados respondieron contar con más de 11 hectáreas de terrenos en producción; bajo este contexto, la persona que se encarga de realizar la mayoría de las actividades administrativas y operativas del negocio es el mismo dueño, dado que la mayoría son negocios de tipo familiar y que se han heredado de manera generacional.

Tabla 6

Concentrado de respuestas

relacionadas con la productividad.

\begin{tabular}{|c|c|c|c|}
\hline Indicadores & Opciones & Frecuencia & Porcentaje \\
\hline \multirow{4}{*}{$\begin{array}{l}\text { Superficie de agave que } \\
\text { cultivan actualmente }\end{array}$} & $4-5$ ha & 16 & $26,7 \%$ \\
\hline & $6-8$ ha & 23 & $38,3 \%$ \\
\hline & $9-10$ ha & 14 & $23,2 \%$ \\
\hline & 11-más & 7 & $11,7 \%$ \\
\hline \multirow{2}{*}{$\begin{array}{l}\text { Gasto hecho desde la } \\
\text { siembra hasta la cosecha }\end{array}$} & $31,000-40,000$ & 1 & $1,7 \%$ \\
\hline & 41,000-más & 59 & $98,3 \%$ \\
\hline \multirow{2}{*}{$\begin{array}{l}\text { Mercado de venta de los } \\
\text { productos (mezcal) }\end{array}$} & Mercado Estatal & 31 & $51,7 \%$ \\
\hline & Mercado Nacional & 29 & $48,3 \%$ \\
\hline \multirow{3}{*}{$\begin{array}{l}\text { Encargado de realizar las } \\
\text { ventas }\end{array}$} & El productor directamente & 17 & $28,3 \%$ \\
\hline & Una comercializadora & 26 & $43,3 \%$ \\
\hline & Puntos de venta del negocio & 17 & $28,3 \%$ \\
\hline \multirow{3}{*}{$\begin{array}{l}\text { Número de personas que } \\
\text { laboran en el negocio }\end{array}$} & 1-10 personas & 40 & $66,7 \%$ \\
\hline & $11-30$ personas & 17 & $28,3 \%$ \\
\hline & 31- más & 3 & $5 \%$ \\
\hline
\end{tabular}

Fuente: Elaboración propia con base en el trabajo de campo (2020).

Por otro lado, es importante mencionar que la comercialización de sus productos lo hacen en el mercado estatal y nacional con el $51.7 \%$ y el $48.3 \%$ respectivamente, y en su caso son entregados a los intermediarios que muchas veces le colocan sus etiquetas para exportarlo a otros países sin el conocimiento de los productores y esto sucede porque los productores argumentan que desconocen los procedimientos que se deben realizar para exportar, por ello les es más fácil entregar su producción a intermediarios que son los que más ganan como sucede en éste y en otros sectores de la economía mexicana.

Este fenómeno se repite en el ámbito de la tecnología: los productores destinan un pequeño porcentaje de sus 
ingresos a la compra de maquinaria y equipo y lo hacen de manera esporádica; los productores encuestados mencionaron que efectivamente no lo hacen porque le cambia el sabor, aroma y la calidad al producto final, además porque pertenecen al área considerada DO y para mantener la denominación deben producir de manera artesanal, por ello afirman que todos los productores de la región producen de manera artesanal, aunque por otro lado están conscientes que si comenzaran a transitar hacia el uso de maquinaria y equipo, eso les ayudaría a incrementar su capacidad de producción y a reducir sus gastos operativos y administrativos; a pesar de ello, todos los encuestados tienen la intención y desean exportar sus productos al mercado internacional.

Tabla 7

Concentrado de resultados

de la variable Tecnología.

\begin{tabular}{lccc}
\hline & Opciones & Frecuencia & Porcentaje \\
\hline Porcentaje del ingreso que invierten en & $\mathbf{5 \%}$ & $\mathbf{5 8}$ & $\mathbf{9 6 . 7 \%}$ \\
\cline { 2 - 3 } maquinaria y equipo & $10 \%$ & 1 & $1,7 \%$ \\
\cline { 2 - 3 } & $15 \%$ & 1 & $1,7 \%$ \\
\hline La última ocasión en que compraron alguna & 1 año & $3,3 \%$ \\
\cline { 2 - 3 } maquinaria o equipo & 2 años & $3,3 \%$ \\
\cline { 2 - 3 } & 3 años & $6,7 \%$ \\
\hline Utiliza computadora para realizar el registro & 4 años & $\mathbf{2}$ & $\mathbf{8 0 . 0 \%}$ \\
de sus operaciones & $\mathbf{4}$ años-más & $\mathbf{1 3 , 3 \%}$ \\
\hline El negocio cuenta con página de internet & Sí & $\mathbf{4 8}$ & $\mathbf{8 6 , 7 \%}$ \\
\hline La mayoría de los productores de la región utilizan & Sí & $\mathbf{5 2}$ & 31.7 \\
\cline { 2 - 3 } maquinaria y equipo para su producción & No & $\mathbf{4 1}$ & $\mathbf{6 8 . 3}$ \\
\hline
\end{tabular}

Fuente: Elaboración propia con base al trabajo de campo (2020)

En lo referente a la variable estructura organizacional, dado que la mayoría de los encuestados tienen negocios de tipo familiar, la persona que toma las decisiones en la empresa es el dueño, por lo tanto no existe una estructura organizacional dentro de la misma, no cuentan con políticas de la empresa que permita a los empleados conocer los alcances y limitaciones que tienen dentro de su área de trabajo; aunado a esto los propietarios de las empresas no contratan servicios de capacitación para su personal, asumen que el personal conoce sus funciones y obligaciones, esto sucede porque no existe un protocolo para la contratación de personal, la mano de obra la consiguen en la misma región y en algunas etapas del proceso de producción mencionan que los desempleados buscan donde ocuparse y en ocasiones se ven obligados a contratar personal de otras regiones para trabajar. Se puede percibir que el nivel de escolaridad que tiene el personal en su mayoría es el nivel básico y en menor medida el nivel medio superior, siendo ésta una de las razones por el que, aún teniendo las condiciones y características para exportar, se ven limitados por la falta de información y de personal con conocimiento que se encargue de todo el proceso de exportación.

Tabla 8

Concentrado de resultados de la variable Estructura Organizacional.

\begin{tabular}{lccc}
\hline & Opciones & Frecuencia & Porcentaje \\
\hline Quién toma las decisiones en la & El dueño & $\mathbf{5 5}$ & $\mathbf{9 1 , 7 \%}$ \\
\cline { 2 - 4 } empresa & El administrador & $3,3 \%$ \\
\cline { 2 - 4 } & El consejo de administración & $5.0 \%$ \\
\hline El personal cuenta con un jefe directo & Sí & $\mathbf{5 3}$ & $\mathbf{8 8 , 3 \%}$ \\
\cline { 2 - 4 } & No & $\mathbf{7}$ & $\mathbf{5 8}$ \\
\hline Cada personal cuenta con funciones & Sí & 2 & $\mathbf{9 6 , 7 \%}$ \\
\hline específicas a realizar & No & $\mathbf{5 7}$ & $3,3 \%$ \\
\hline La empresa cuenta por escrito sus & Sí & 3 & $\mathbf{9 5 \%}$ \\
planes a corto, mediano y largo plazo & No & $5 \%$ \\
\hline
\end{tabular}




\begin{tabular}{lccc}
\hline & Opciones & Frecuencia & Porcentaje \\
\hline El personal recibe capacitación & Sí & 15 & $25 \%$ \\
\hline & No & $\mathbf{4 5}$ & $\mathbf{7 5 \%}$ \\
\hline $\begin{array}{l}\text { La empresa cuenta con un } \\
\text { organigrama }\end{array}$ & Sí & $\mathbf{2}$ & $3,3 \%$ \\
\hline $\begin{array}{l}\text { Medio por el que realizan la } \\
\text { contratación de los trabajadores }\end{array}$ & No & $\mathbf{5 8}$ & $\mathbf{9 6 , 7 \%}$ \\
\cline { 2 - 4 } & Por entrevista & 11 & $18,3 \%$ \\
& Por recomendación & $\mathbf{4 9}$ & $\mathbf{8 1 , 7 \%}$ \\
\hline
\end{tabular}

Fuente: Elaboración propia con base en el trabajo de campo (2020).

En la siguiente tabla se muestra la prueba de coeficiente de correlación de Pearson, en el que se observa una alta correlación entre la variable productividad y tecnología con un .459 , no así con la variable estructura organizacional con -.411, considerando que si el coeficiente es igual a -1 nos referimos a una correlación negativa perfecta; por el contrario, si el coeficiente es igual a +1 , existe una correlación positiva perfecta. Con esta herramienta estadística se comprueba la incidencia negativa que existe de las variables independientes con la variable dependiente, que es la competitividad exportadora de las MIPYMES productoras de mezcal en las entidades objeto de estudio.

Tabla 9

Coeficiente de correlación de Pearson

\begin{tabular}{|c|c|c|c|c|}
\hline Variables & & Productividad & Tecnología & Estructura Organizacional \\
\hline \multirow[t]{3}{*}{ Productividad } & Correlación de Pearson & 1 & $.459 * *$ & $-.411 * *$ \\
\hline & Sig. (bilateral) & & .000 & .000 \\
\hline & $\mathrm{N}$ & 100 & 100 & 100 \\
\hline \multirow[t]{3}{*}{ Tecnología } & Correlación de Pearson & $.459 * *$ & 1 & $-.235^{*}$ \\
\hline & Sig. (bilateral) & .000 & & .018 \\
\hline & $\mathrm{N}$ & 100 & 100 & 100 \\
\hline \multirow[t]{3}{*}{ Estructura Organizacional } & Correlación de Pearson & $-.411 * *$ & $-.235^{*}$ & 1 \\
\hline & Sig. (bilateral) & .000 & .018 & \\
\hline & $\mathrm{N}$ & 100 & 100 & 100 \\
\hline
\end{tabular}

Fuente: Elaboración propia con base en el trabajo de campo (2020).

Para conocer el comportamiento de las variables independientes sobre la variable dependiente y comprobar la hipótesis planteada, así como la revisión literaria realizada, se aplicó a los datos de trabajo de campo la prueba de causalidad de Granger para cada una de las variables obteniendo los siguientes resultados.

Como se observa en la tabla 10 el bloque de valores rezagados de la Productividad es estadísticamente significativo, bajo el supuesto que para ser significativo debe reflejar una cantidad menor a 0.05, por lo tanto, en este caso afecta a la Competitividad de manera significativa, se puede afirmar que el nivel de competitividad está en función de la Productividad. 
Tabla 10

Prueba de causalidad de Granger de la variable Productividad

\begin{tabular}{|c|c|c|c|}
\hline \multicolumn{5}{|c|}{ VAR Granger Causality/Block Exogeneity Wald Tests } \\
\hline \multicolumn{4}{|c|}{ Date: 02/25/21 Time: 22:01 } \\
\hline Sample: & 115 & \\
\hline \multicolumn{4}{|c|}{ Included observations: 13} \\
Dependent variable: Y_COMPETITIVIDAD \\
\hline Excluded & Chi-sq & Df & Prob. \\
\hline X1_PRODUCTIVIDAD & 7.997746 & 2 & 0.0183 \\
\hline All & 7.997746 & 2 & 0.0183 \\
\hline Dependent variable: X1_PRODUCTIVIDAD \\
\hline Excluded & Chi-sq & Df & Prob. \\
\hline Y_COMPETITIVIDAD & 0.856659 & 2 & 0.6516 \\
\hline All & 0.856659 & 2 & 0.6516 \\
\hline
\end{tabular}

Fuente: Elaboración propia con base en Eviews (2021).

\section{Tabla 11}

Prueba de causalidad de Granger de la variable Tecnología.

\begin{tabular}{|c|c|c|c|}
\hline VAR Granger Causali & ck Exogen & ald & \\
\hline Date: 02/25/21 Time & & & \\
\hline Sample: 115 & & & \\
\hline Included observation & & & \\
\hline Dependent variable: & MPETITIV & & \\
\hline Excluded & Chi-sq & Df & Prob. \\
\hline X2_TECNOLOGIA & 9.482016 & 2 & 0.0087 \\
\hline All & 9.482016 & 2 & 0.0087 \\
\hline Dependent variable: & ECNOLOG & & \\
\hline Excluded & Chi-sq & Df & Prob. \\
\hline Y_COMPETITIVIDAD & 33.09075 & 2 & 0.0000 \\
\hline All & 33.09075 & 2 & 0.0000 \\
\hline
\end{tabular}

Fuente: Elaboración propia con base en Eviews (2021).

Para el caso de la variable Tecnología en la tabla anterior, el bloque de valores rezagados dela Tecnología es estadísticamente significativo, por lo tanto, en este caso afecta a laCompetitividad de manera significativa, se puede afirmar que el nivel de competitividad estáen función de la Tecnología, de igual manera se confirma la hipótesis planteada en este trabajo de investigación.

Para el caso de la variable Estructura Organizacional de la tabla anterior, el bloque de valores rezagados de la variable es estadísticamente significativo, por lo tanto, en este caso afecta a la Competitividad de manera significativa, no en la misma proporción como lo hacen las variables Productividad y Tecnología, sin embargo, sigue siendo significativa, de ahí que se puede afirmar que el nivel de competitividad está en función de la Estructura Organizacional, de igual manera se confirma la hipótesis planteadaen este trabajo de investigación. 
Tabla 12

Prueba de causalidad de Granger de la variable Estructura Organizacional

\begin{tabular}{|c|c|c|c|}
\hline \multicolumn{4}{|c|}{ VAR Granger Causality/Block Exogeneity Wald Tests } \\
\hline \multicolumn{4}{|c|}{ Date: 02/25/21 Time: 22:05 } \\
\hline \multicolumn{4}{|l|}{ Sample: 115} \\
\hline \multicolumn{4}{|c|}{ Included observations: 13} \\
\hline \multicolumn{4}{|c|}{ Dependent variable: Y_COMPETITIVIDAD } \\
\hline Excluded & Chi-sq & Df & Prob. \\
\hline X3_ESTRUCTURA_ & 7.891230 & 2 & 0.0193 \\
\hline \multicolumn{4}{|l|}{ ORGANIZACIONAL } \\
\hline All & 7.891230 & 2 & 0.0193 \\
\hline \multicolumn{4}{|c|}{ Dependent variable: X3_ESTRUCTURA_ORGANIZACIONAL } \\
\hline Excluded & Chi-sq & Df & Prob. \\
\hline Y_COMPETITIVIDAD & 2.531027 & 2 & 0.2821 \\
\hline All & 2.531027 & 2 & 0.2821 \\
\hline
\end{tabular}

Fuente: Elaboración propia con base en Eviews (2021)

\subsection{Discusión}

De acuerdo a Larios (2016), en México existen poco más de 4, 000,000 de empresas y sólo 6, 700 son empresas grandes, el resto son MIPYMES y representan el $99.8 \%$, generan $52 \%$ del PIB y contribuyen con el $72 \%$ de los empleos formales. Sin embargo; es el sector más castigado en cuanto a la falta de apoyos, entendido éste como financiamiento público y privado, asesoramiento, capacitación, vinculación con clientes potenciales a nivel nacional e internacional, de ahí que un porcentaje muy reducido del total de las MIPYMES en México se dedican a la exportación de sus bienes y servicios hacia otros países.

Esta marginación que viven las MIPMYMES, es más marcado aún en las actividades relacionadas con el sector primario, de ahí que los productores de mezcal padecen de estas limitaciones históricamente, es predecible que sigan así dado que los esfuerzos que han hecho de manera aislada como sector, no han logrado obtener los beneficios esperados, esto no es por la falta de calidad de sus productos, ni por desconocimiento de los productores para generar los productos, esta problemática está directamente relacionada con aspectos normativos, legales, y de control por parte de las instancias que regulan la actividad.

\section{Conclusiones}

De acuerdo a los datos estadísticos analizados, se pueden probar las hipótesis planteadas al inicio de esta investigación en el sentido que las variables Productividad, Tecnología y Estructura Organizacional tienen una incidencia significativa en la competitividad de las MIPYMES productoras de mezcal en los estados objeto de estudio, razón principal por el que aun teniendo todos los elementos y capacidad, siguen inmersos en el mercado estatal y nacional sin dar el siguiente paso para exportar directamente sus productos. Esto no significa que el mezcal no tenga calidad de exportación, en la mayoría de los casos sí lo tiene, pero son realizados por los intermediarios, que se llevan la mayor ganancia aprovechando el desconocimiento de los productores de este sector. Por ello se propone que los productores se organicen y operen bajo la figura legal de Sociedad de Producción Rural (SPR) para aprovechar sus fortalezas y generar un sólo esquema de comercialización y exportación de su producto.

\section{Referencias bibliográficas}

Bautista, J. (2015). La disminución de la producción artesanal de mezcal en la Región del mezcal de Oaxaca, México. . Revista Mexicana Ciencias Agrícolas, vol. 6, no. 6. 
Chávez, E. (2012). Competitividad e innovación tecnológica como factores de cambio institucional, retos y oportunidades para México. XVII Congreso Internacional de Contaduría Administración e Informática., pp. 1-18.

Chávez, M. (2016). Revisión del Agave y del Mezcal. Revista Colombiana Biotenologo, vol. XVIII, no. 1, pp 148164.

Claver, E. (2000). La tecnología como factor de competitividad: un análisis a través de la teoría de recursos y capacidades. Boletín de Estudios Económicos . v. 5, no. 169, pp. 119-138.

Esser, Hilebrand, Messner y Meyer. (1994.). Competitividad sistémica. Instituto Alemán de Desarrollo, Berlín. rong Democracy. Participatory Politics for a New Age, Berkeley 1984. Capítulo IV.

Hintze, J. (1999). Administración de estructuras organizativas. Centro de Desarrollo y asistencia Técnica en Tecnología para la Organización Pública TOP, 17. Centro de Desarrollo y Asistencia Técnica en Tecnología para organización Pública TOP, Buenos Aires.

Ibarra, M. (2017). Competitividad empresarial de las pequeñas y medianas empresas manufactureras de Baja California. Estudios Fronterizos, 107-130, no. 18.

Idárraga, M. (2012). Estructura organizacional y sus parámetros de diseño: análisis descriptivo en pymes industriales de Bogotá. Estudios Gerenciales, Vol. 28, no. 123, pp. 43-63.

Jaimes, Ludym. (2018). Factores determinantes de la productividad laboral en pequeñas y medianas empresas de confecciones del área metropolitana de Bucaramanga, Colombia. Vo. 29, No. 5, p.p. 175-186.

Jinémez, M. (2006). Modelo de competitividad empresarial. Umbral Científico, no. 9, pp 115-125.

María Saavedra, Blanca Tapia. (2011). Mejores prácticas y factores de competitividad en las micro, pequeñas y medianas empresas mexicanas. Economía, 11-36, no. 32.

Matta, C. (2015). La innovación Tecnológica como ventaja competitiva en las empresas. Seminario de Innovación Empresarial y Emprendimiento por la Universidad Autónoma de Barcelona. , pp. 1-19.

Mintzberg, H. (1993). El proceso estratégico conceptos, contextos y casos. Prentice Hall Hispanoamericana, 18.

Nagles, G. (2007). Gestión del Conocimiento como fuente de Innovación. Escuela de Administración de Negocios, pág. 77-87, no. 61. Universidad EAN, Bogotá, Colombia.

Pérez, E. (2016). Revisión del agave y el mezcal. Revista Colombiana de Biotecnología, Vol. XVIII, no. 1, pp. 148164.

Pérez, R. (2007). Estructura y cultura Organizacional en la Pyme Colombiana: Análisis en empresas Bogotanas. Cuadernos de Administración , 73-85, no. 38.

Placencia; Peralta. (2018). Análisis histórico de los mezcales y su situación actual desde una perspectiva ecomarxista. . Revista de Desarrollo Económico Territorial., no. 14, pp. 20.

Porter, M. (2015). Estratégia competitiva. México. Editorial Patria. 
Ríos, M. (2014). Análisis de la preparación tecnológica en las pymes. Revista Internacional Administración y Finanzas. , vol. 7, no. 7 pp. 16.

Rugman, A. (2001). Negocios Internacioales: Un enfoque de administración estratégica. México: McGraw Hill. Pág. 235. Universidad de Guadalajara.

Saldaña, I. (2012). Anatomía del mezcal. pp. 5. Recuperado de: http://www.anatomiadelmezcal.com/\#!/home

Víctor Alvarado, Ana Sánchez. (2019). Propuesta de un modelo para la medición aproximada de la productividad. Retos de las ciencias administrativas desde las economías emergentes. Evolución de sociedades. Pág. 15. Tecnológico de Monterrey, Instituto politécnico Nacional. Cuidad de México, México.

\section{Anexo 1. Cuestionario aplicado en campo}

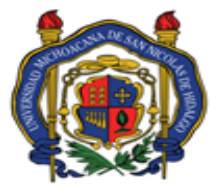

Universidad Michoacana de San Nicolás de Hidalgo

Instituto de Investigaciones Económicas y Empresariales

\section{Cuestionario}

El objetivo de este cuestionario es determinar cuáles han sido las principales variables que han incidido en la competitividad exportadora de las Micro Pequeñas y Medianas Empresas (MIPYMES) productoras de mezcal ubicadas en los municipios de Santiago Matatlán y Santa Catarina Minas del estado de Oaxaca; y Villa Madero y Queréndaro del estado de Michoacán, México, hacia el mercado de Estados Unidos.

Instrucciones: Marque con una $\mathbf{X}$ la respuesta a cada pregunta que considere correcta de acuerdo a su experiencia y conocimiento sobre el proceso de producción y comercialización de mezcal. Esta encuesta es realizada con fines académicos y su participación será anónima.

Estado:

Nombre del Municipio:

Nombre de la empresa:

No. De trabajadores:

Puesto que ocupa en la empresa:

\section{Productividad}

1. ¿En cuánta superficie (hectárea) cultiva actualmente agave para la producción de mezcal?
a) 1 a 3
b) 4 a 5
c) 6 a 8
d) 9 a 10
e) más de 11 
2. ¿Cuántos kilos de agave produce en 1 hectárea?
a) 20,000 a 30,000
b) 31,000 a 40,000
c) 41,000 a 50,000
d) más de 51,000

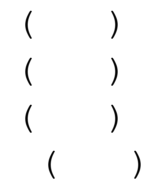

3. ¿Cuántos kilos de agave utiliza para producir 1 litro de mezcal?
a) 5 a 7
b) 8 a 10
c) 11 a 13
d) más de 14

4. ¿Cuánto dinero gasta para producir una hectárea de agave desde la siembra hasta la cosecha?
a) 10,000 a 20,000
b) 21,000 a 30,000
c) 31,000 a 40,000
d) más de 41,000

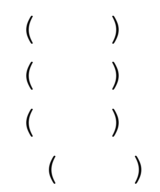

5. ¿Desde hace cuántos años se dedica a la producción de mezcal?
a) 5 a 10
b) 11 a 15
c) 16 a 20
d) 21 a 25
e) más de 26

6. ¿La totalidad de la producción que obtiene en dónde lo vende?
a) Mercado local
b) Mercado estatal
c) Mercado Nacional
d) Mercado Internacional

7. ¿Ha considerado la posibilidad de vender sus productos de manera directa a otros países?
a) $\mathrm{Si}$
b) No

8. ¿La venta del mezcal quién la realiza?

a) El productor

b) Una empresa revendedora ( )

c) Negocios pequeños

d) Otros 
9. ¿Cuántas personas laboran en este negocio?
a) 1 a 5
b) 6 a 10
c) 11 a 15
d) 16 a 20
e) más de 21

10. ¿Cuántas horas laboran los trabajadores al día?
a) 6 a 8
b) 9 a 10
c) 11 a 12
d) más de 12

11. ¿Cuánto se le paga a un trabajador de manera semanal?
a) $\$ 500$ a $\$ 800$
b) $\$ 801$ a $\$ 1,000$
c) $\$ 1,001$ a $\$ 1,200$
d) $\$ 1,201$ a $\$ 1,500$
e) más de 1,500

12. ¿El negocio cubre sus gastos con los ingresos que obtiene?
a) Sí
b) No
c) más o menos

13. ¿Paga renta por las tierras donde produce?
a) Sí
b) No

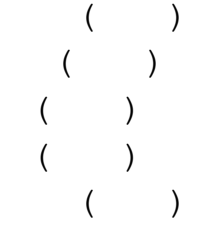

\section{Tecnología}

14. ¿Qué porcentaje del ingreso de la empresa lo invierte en la compra de maquinaria y equipo?
a) $5 \%$
b) $10 \%$
c) $15 \%$
d) $20 \%$
e) $25 \%$

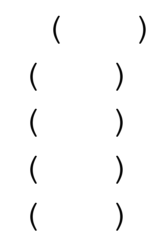

15. ¿Desde hace cuánto tiempo compró alguna maquinaria o equipo para la producción de mezcal?
a) 1 año
b) 2 años
c) 3 años 
d) 4 años

e) más de 4 años

16. ¿La producción de mezcal que obtiene lo hace de manera artesanal - con el uso de alguna máquina?
a) Máquina

b) Artesanal

17. ¿Considera que es más rápido producir mezcal con la ayuda de una máquina?
a) $\mathrm{Si}$
b) No

18. ¿Ha logrado cubrir en tiempo y forma a sus clientes en la entrega de los productos?
a) $\mathrm{Si}$
b) No

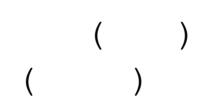

19. ¿Ha considerado la posibilidad de producir una cantidad mayor a la que actualmente produce?

a) Si

20. ¿El registro de sus operaciones diarias lo realiza con la ayuda de una computadora?

a) $\mathrm{Si}$

b) No

21. ¿En orden de importancia a través de qué medio realiza sus ventas? (En donde 1 es de mayor importancia y 4 de menor importancia)

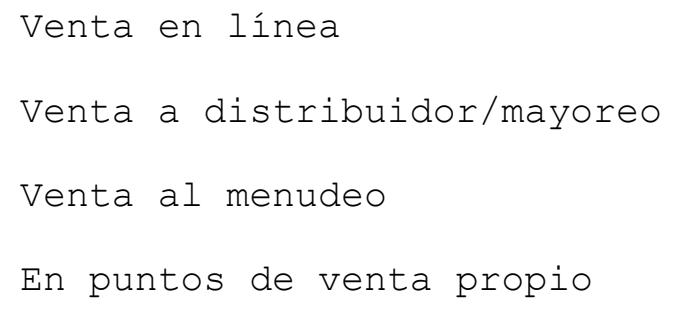
a) $\mathrm{Si}$
b) No

$(1)$


23. ¿La compra de máquinas y equipos para producir generan un mayor costo para el negocio?
a) $\mathrm{Si}$
b) No

24. ¿Cómo considera para el negocio la compra de más máquinas para producir mezcal?
a) Bueno ( )
b) Malo

25. ¿La mayoría de los productores de la región utilizan maquinaria para producir mezcal?
a) Sí
b) No

26. ¿A quiénes les va mejor? A los que producen de manera artesanal - a los que lo hacen con la ayuda de maquinaria.
a) Los que producen de manera artesanal
b) Los que producen con ayuda de máquinas

\section{Estructura Organizacional}

27. ¿Quién toma las decisiones en la empresa?
a) El dueño
b) El administrador
c) El consejo de administración

28. ¿Cada personal tiene un jefe directo?
a) $\mathrm{Si}$
b) No

29. ¿Quién se encarga de la contratación del personal?
a) El dueño
b) El área de recursos humanos
c) El administrador

30. ¿La empresa cuenta con metas a alcanzar a corto, mediano y largo plazo?
a) $\mathrm{Si}$
b) No

31. ¿Se le pide opinión a los trabajadores para mejorar la operación/administración de la empresa?
a) $\mathrm{Si}$
b) No 
32. ¿La empresa tiene por escrito sus reglamentos internos, que quiere alcanzar en un futuro y como lograrlo?
a) $\mathrm{Si}$
b) No

33. ¿El personal cuenta con posibilidades de crecimiento dentro de la empresa?

a) $\mathrm{Si}$

34. ¿Cada persona realiza una actividad en específico dentro de la empresa?
a) $\mathrm{Si}$
b) No

$\left(\begin{array}{ll}( \\ (\end{array}\right)$

35. ¿El personal recibe capacitación para mejorar su desempeño?
a) $\mathrm{Si}$
b) No

36. ¿La empresa cuenta con un documento donde se menciona el puesto y las funciones de cada trabajador?
a) $\mathrm{Si}$
b) No

37. ¿El sueldo de cada trabajador está determinada de acuerdo a su función dentro de la empresa?
a) $\mathrm{Si}$
b) No

38. ¿Existe un reglamento que deben cumplir todos los trabajadores en el negocio?
a) $\mathrm{Si}$
b) No

39. ¿A través de que medio realizan la contratación de trabajadores?
a) Entrevista
( )
b) Por recomendación ( )
c) Anuncio en internet ( )

\section{Datos de identificación}

Muchas gracias por su cooperación y tiempo brindado al presente cuestionario. 


\section{Datos del entrevistado:}

Fecha de la entrevista

Nombre del entrevistado

Sexo

Ocupación

Escolaridad

Dirección (Opcional)

Colonia

Teléfono (Opcional)

Correo electrónico

\section{(c) $(1)(8)$}

Esta obra está bajo una Licencia Creative Commons

Atribución-NoComercial 4.0 Internacional 